\title{
Non-Symmetrical Bis-Azine Biaryls from Chloroazines: A Strategy Using Phosphorus Ligand-Coupling
}

\author{
Benjamin T. Boyle ${ }^{\circledR}$, Michael C. Hilton ${ }^{\circledR}$ and Andrew McNally*(D) \\ Department of Chemistry, Colorado State University, Fort Collins, Colorado 80523, United States
}

\section{Supporting Information Placeholder}

\begin{abstract}
Distinct approaches to synthesize bis-azine biaryls are in demand as these compounds have multiple applications in the chemical sciences and are challenging targets for metal-catalyzed cross-coupling reactions. Most approaches focus on developing new reagents as the formal nucleophilic coupling partner that can function in metal-catalyzed processes. We present an alternative approach using pyridine and diazine phosphines as nucleophilic partners and chloroazines where the heterobiaryl bond is formed via a tandem $\mathrm{S}_{N} \mathrm{Ar}$-phosphorus ligand-coupling sequence. The heteroaryl phosphines are prepared from chloroazines and are bench stable solids. Using this strategy, a range of bis-azine biaryls can be formed from abundant chloroazines that would be challenging using traditional approaches and a one-pot cross-electrophile coupling of two chloroazines is feasible.
\end{abstract}

Bis-azine biaryls are widely applied in the chemical sciences with applications in drug development, as ligands for metal complexes and as components of materials (eq 1). ${ }^{1}$ Transition metal catalyzed cross-coupling reactions are most commonly used to synthesize non-symmetrical variants, however, pyridine and diazine couplings represent some of the most challenging cases for this class of reactions. ${ }^{2}$ Readily available and stable cross-coupling precursors are often limiting factors; haloazines are relatively abundant from commercial sources compared to nucleophilic partners such as pyridyl zincs, stannanes, silanes and Grignards that often have to be prepared and carefully handled. ${ }^{3}$ Suzuki-Miyaura reactions are the most widely applied in medicinal chemistry as azine boronic acids are typically bench-stable powders. ${ }^{4}$ Despite this advantage, halopyridines remain dramatically more commercially available than pyridine boronic acids, and 2-substituted pyridine boronic acids have a proclivity to decompose during cross-coupling reactions. ${ }^{5,6}$ Herein we show that azinylphosphines can serve as nucleophilic partners and couple with haloazines where the biaryl bond is formed via a phosphorus ligand-coupling reaction. ${ }^{7}$ These phosphines are straightforward to prepare from haloazines and are bench-stable. Preliminary examples of a net cross-electrophile coupling are shown via a one-pot coupling of two heteroaryl halides.

Several groups have developed alternatives to azine boronic acids for metal-catalyzed cross-coupling reactions. Potassium trifluoroborate salts and MIDA boronates display enhanced stability compared to boronic acids, but a general platform for azine-azine coupling has yet to be established using these reagents. ${ }^{8}$ Willis advanced this area by demonstrating that pyridyl sulfinate salts and allyl sulfones are viable alternatives for Suzuki couplings. ${ }^{9} \mathrm{Re}-$ cently, we reported an alternative strategy to form bis-azine biaryls by constructing bis-azine phosphonium salts from $\mathrm{C}-\mathrm{H}$ precursors and then exploiting phosphorus ligand-coupling reactions to form

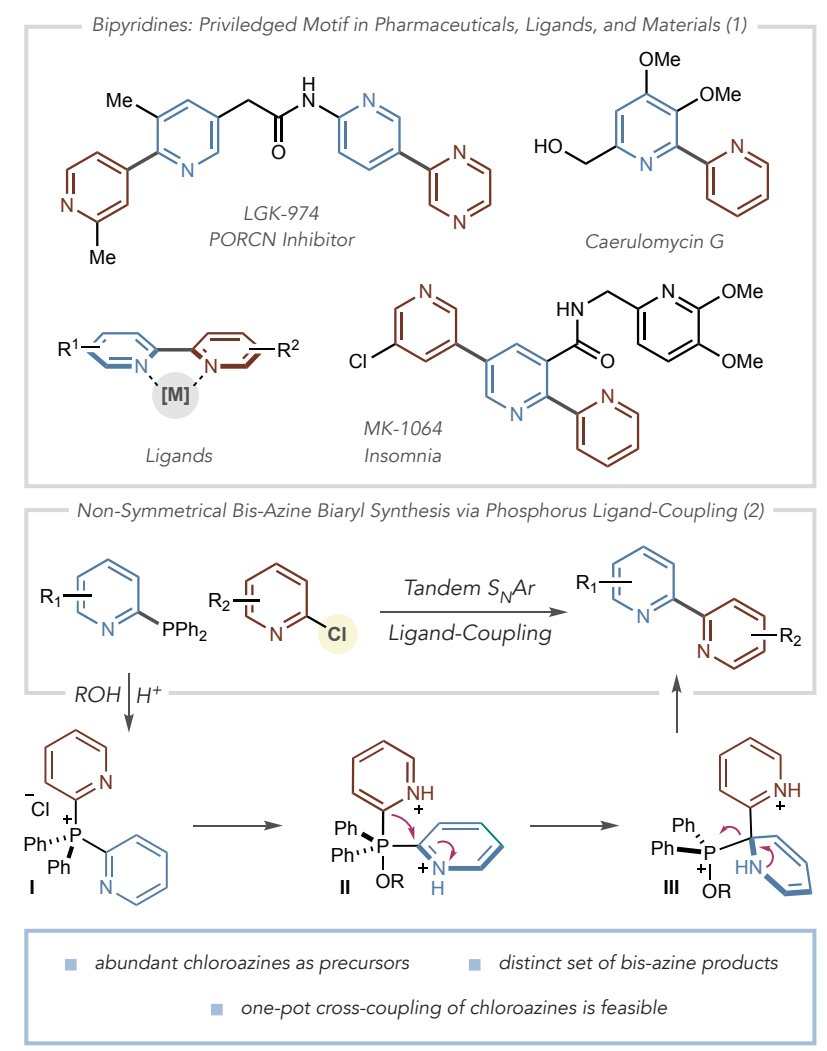

the biaryl bond ${ }^{10}$ However, the inherent selectivity of the two C-P bond-forming steps restrict access to bis-azine biaryls within some isomeric patterns such as 2,2'-bipyridines (vide infra). Furthermore, certain functional groups and azine substitution patterns are not tolerated. We envisioned a strategy analogous to metal-catalyzed cross-coupling using chloroazines and heteroaryl phosphines as precursors (eq 2). This approach could overcome limitations of current metal-catalyzed processes as well as result in a suite of bisazine biaryls that are beyond the scope of our original report. Pyridyl and diazinyl phosphines, prepared in one step from chloroazines, can function as nucleophiles and couple with a second chloroazine via a tandem $\mathrm{S}_{N} \mathrm{Ar}$-ligand-coupling sequence; the mechanism involves forming a bis-heteroarylphosphonium salt (I) that is intercepted in acidic alcohol or aqueous solutions to form a $\mathrm{P}(\mathrm{V})$ alkoxyphosphorane intermediate (II). In line with our previous studies, we anticipated that subsequent ligand-coupling would proceed via an asynchronous process, involving a dearomatized species III as a discrete intermediate. 


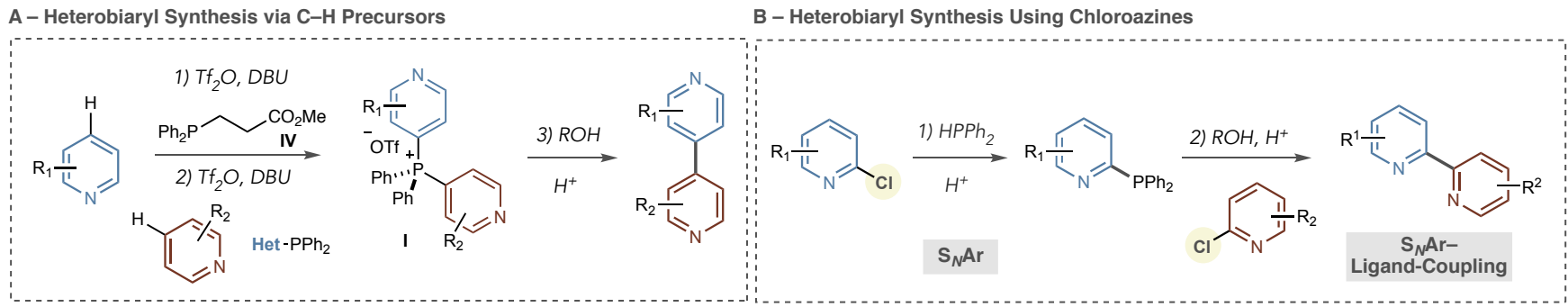

C - Limitations of the Coupling Strategy Using C-H Precursors and Alternative Chloroazines

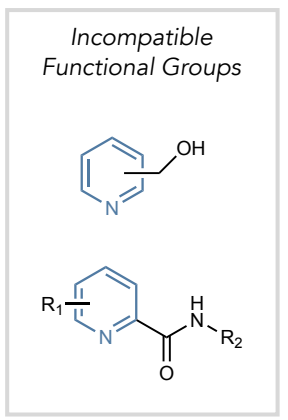

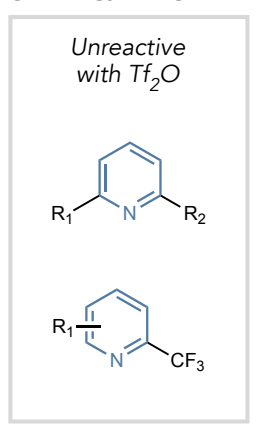

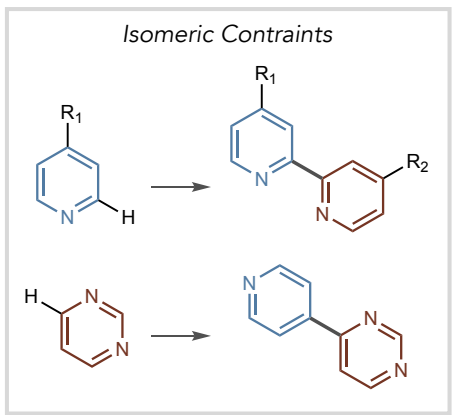

Solution - $S_{N}$ Ar Reactions with Abundant Cholorazines

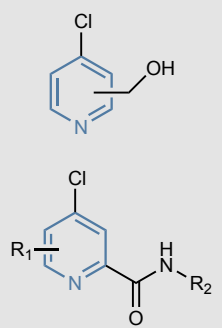<smiles>Clc1ccncc1</smiles><smiles>[R]=Cc1cc(Cl)cc(C(F)(F)F)n1</smiles>

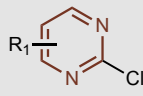

Scheme 1 shows specific limitations of our previous approach using azine $\mathrm{C}-\mathrm{H}$ bonds as precursors and potential solutions using chloroazines. In the former case, two $\mathrm{C}-\mathrm{P}$ bond-forming reactions are required to form bis-heterocyclic phosphonium salt I (Scheme 1A). Fragmentable phosphine IV is used with $\mathrm{Tf}_{2} \mathrm{O}$ and DBU as a base to form an azinylphosphine (not shown); the process is then repeated with the second azine coupling partner to form salt I. Under these conditions, common functional groups such as alcohols, phenols and alkyl-substituted amides, ${ }^{11,12}$ are not tolerated due to their propensity to react with $\mathrm{Tf}_{2} \mathrm{O}$ (Scheme 1C). Pyridines with certain substitution patterns are also not amenable; 2,6-disubstituted pyridines and $2-\mathrm{CF}_{3}$ pyridines are unsuccessful as the $s p^{2}$ nitrogen atom is either too sterically crowded or reduced in nucleophilicity such that reaction with $\mathrm{Tf}_{2} \mathrm{O}$ is ineffective. For pyridines and quinolines, both $\mathrm{C}-\mathrm{P}$ bond-forming reactions are inherently 4 selective, whereas 2-position selectivity can only be achieved when the 4-position is blocked. Using this sequence to prepare 2,2'-bipyridines for example, 4-position substituents must be present to ensure the correct positional selectivity. Similarly, C-P bond-formation in diazines is also inherently selective; pyrimidines react at the 4-position in this manifold, and this selectivity restricts the possible isomeric patterns in the resulting bis-azine biaryl. The strategy in Scheme 1B can potentially overcome these issues by using $\mathrm{S}_{N} \mathrm{Ar}$ reactions with chloroazines for each $\mathrm{C}-\mathrm{P}$ bond-forming event. Scheme 1C shows classes of readily available chloroazines where the previous functional group incompatibilities and regiomeric constraints can be addressed.

We first developed conditions for $\mathrm{S}_{N} \mathrm{Ar}$ couplings between chloroazines and $\mathrm{HPPh}_{2}$ and intentionally prepared heteroarylphosphines that were precluded from our previous study by steric, electronic or isomeric constraints. ${ }^{10}$ For 2- and 4-chloropyridines, heating in chlorobenzene at $130{ }^{\circ} \mathrm{C}$ with one equivalent of TfOH was effective, and pyridylphosphines $\mathbf{2} \mathbf{a}-\mathbf{2} \mathbf{g}$ were formed in high yields (condition set $\mathrm{A}$ ) and addresses the aforementioned restrictions in $\mathrm{C}-\mathrm{P}$ bond formation (vide supra). For more $\mathrm{S}_{N} \mathrm{Ar}$ active substrates, such as chloroquinolines, chloroisoquinolines and chlorodiazines, ${ }^{13}$ trifluoroethanol (TFE) at $80^{\circ} \mathrm{C}$ was sufficient and acid activation is not required (condition set $\mathrm{B}$ ). Notably, these conditions allow access to phosphines at the 2-position of quinolines and pyrimidines. We found that these heteroarylphosphines are bench-stable but, as a precaution, they were kept in a $-20{ }^{\circ} \mathrm{C}$ refrigerator for long-term storage.

With a set of heterocyclic phosphines in hand, we next developed one-pot procedures to form bis-azine biaryls via the tandem $\mathrm{S}_{N} \mathrm{Ar}-$ ligand-coupling sequence (Table 2). We found that coupling reactions could be separated into three categories depending on the ease

Table 1. $\mathrm{S}_{N} \mathrm{Ar}$ Reactions to Form Heteroarylphosphines ${ }^{a}$
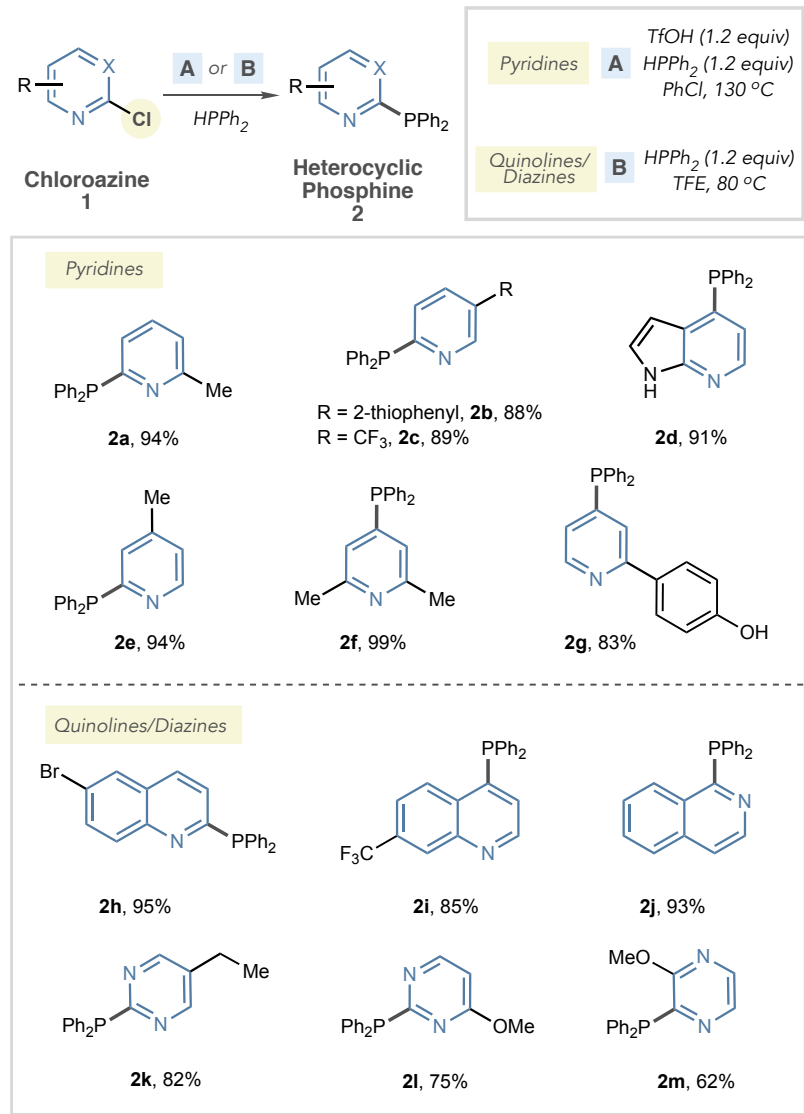

${ }^{a}$ Isolated yields are reported. 
of the $\mathrm{S}_{N} \mathrm{Ar}$ process. First, 2,4-and 4,4'-bipyridines were synthesized in a two-stage process; heating the phosphine and chloropyridine in dioxane at $120^{\circ} \mathrm{C}$ with one equivalent each of $\mathrm{HCl}$ and NaOTf drives the $\mathrm{S}_{N} \mathrm{Ar}$ reaction to completion and forms the bisheterocyclic phosphonium salt. ${ }^{14}$ Then, a further equivalent of $\mathrm{HCl}$, ten equivalents of $\mathrm{H}_{2} \mathrm{O}$, and TFE are added, and heating at $80^{\circ} \mathrm{C}$ is optimal for the ligand-coupling process (condition set $\mathrm{A}^{\prime}$ ). Without $\mathrm{NaOTf}$, the $\mathrm{S}_{N} \mathrm{Ar}$ reaction does not reach full conversion, and we presume that bis-azine phosphonium salt formation is promoted by anion exchange and precipitation of $\mathrm{NaCl}$. By employing these conditions, a set of 2,4- and 4,4'- bipyridines are formed in reasonable yields (3a-3e). Bipyridine $\mathbf{3 b}$ is notable as the alkyl amide group was incompatible with our previous protocol. Second, chloroquinolines and chlorodiazines couple heteroarylphosphines using single-stage protocol. In line with observations in Table 1, these heterocycles undergo more facile $\mathrm{S}_{N} \mathrm{Ar}$ with processes and the tandem $\mathrm{S}_{N} \mathrm{Ar}$-ligand-coupling sequence is performed in TFE at $80^{\circ} \mathrm{C}$ with $\mathrm{H}_{2} \mathrm{O}, \mathrm{HCl}$ and $\mathrm{NaOTf}$ as additives (condition set B'). By employing these conditions, a set of 2,4- and 4,4- pyridine-quinolines are formed (3f-3l) and include examples of pyridines with 2,6-disubstitution, 4-position $\mathrm{C}-\mathrm{H}$ bonds, as well as products containing phenols and alcohols. Furthermore, 2,2-pyridine-quinoline systems 3m-3o can also be synthesized that are challenging to prepare via Suzuki-Miyaura couplings (vide infra). Similarly, $\mathbf{3 p}$ is a

Table 2. Tandem $\mathbf{S}_{\mathbf{N}}$ Ar-Ligand-Coupling Reactions to Form Non-Symmetrical Bis-Azine Biaryls ${ }^{a}$

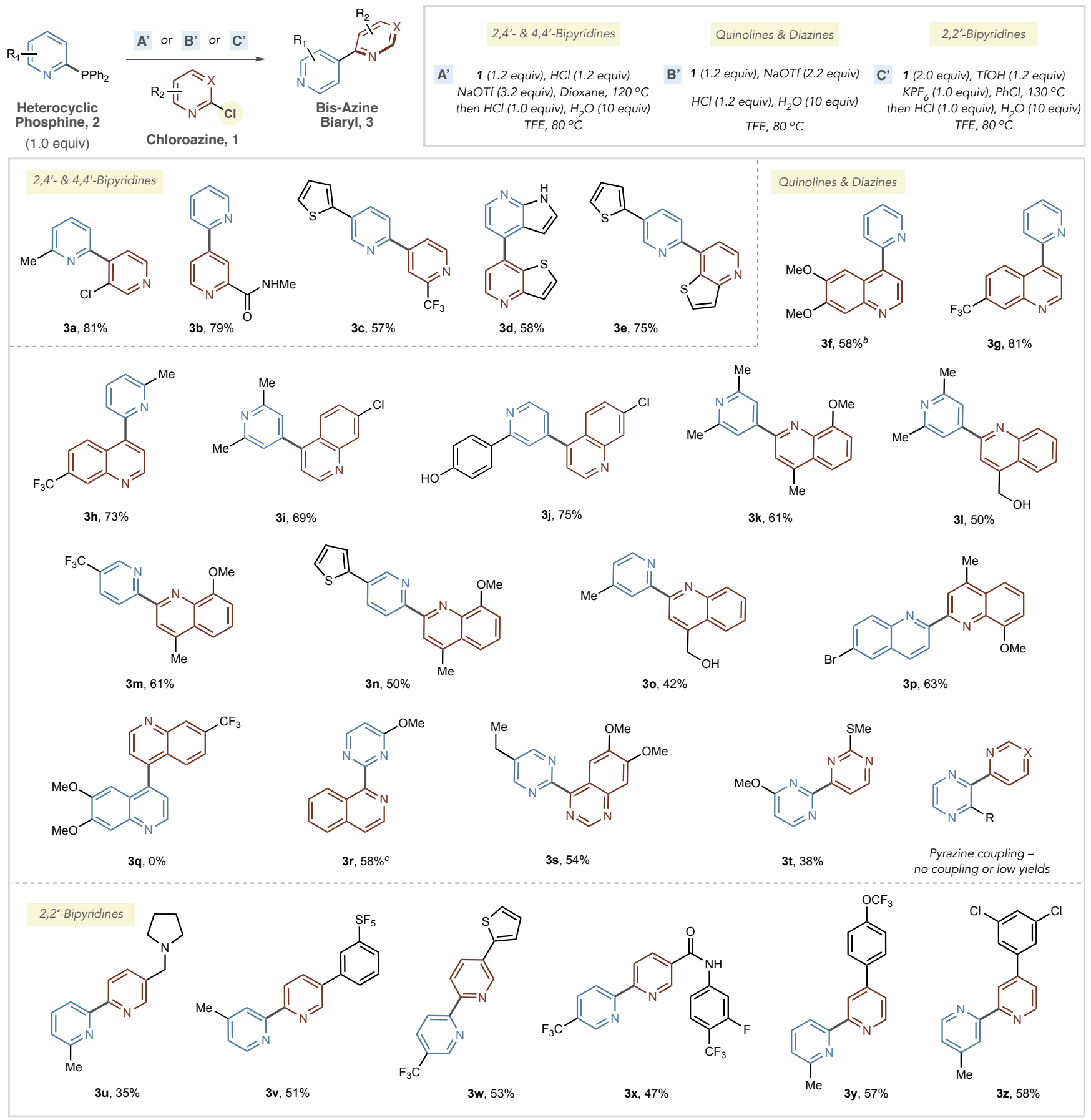

${ }^{a}$ Isolated yields are reported. ${ }^{b} \mathbf{3 f}$ was formed using a two-stage protocol: 1.2 equiv chloroazine, 2.2 equiv $\mathrm{NaOTf}$, TFE, $80{ }^{\circ} \mathrm{C}$ then 1.2 equiv $\mathrm{HCl}$ and 10 equiv water were added. ${ }^{c} \mathrm{TfOH}$ used instead of $\mathrm{HCl}$. 
2,2'-biquinoline that includes a $\mathrm{C}$ - $\mathrm{Br}$ bond that would typically be active in transition metal-catalyzed processes. However, an attempt to form 4,4'-biquinoline $\mathbf{3 q}$ failed; we presume unfavorable steric interactions occur in the ligand-coupling transition state in this case. Diazines can be used as both formal donor phosphines and acceptor chlorides; a 2-pyrimidylphosphine was coupled with a chloroisoquinoline in moderate yield (3r), and diazine-diazine coupling is possible using this approach (3s-3t). At this point pyrazines perform poorly as coupling partners; a short survey of pyridine, quinoline and diazine partners resulted in no coupled products or poor yields (see the Supporting Information). Third, we targeted 2,2'-bipyridines using this strategy due to the challenges in synthesizing these systems using Suzuki-Miyaura cross-coupling reactions (vide supra). Condition set A' was modified to C', where changes in solvent, acid and additive resulted in higher yields of bipyridine products (see Supporting Information). Examples $\mathbf{3 u - 3 z}$ display a variety of isomeric patterns, as well as substituents such as aliphatic amines, $\mathrm{SF}_{5}$ groups, thiophenes, amides and chlorides. As with all other examples in Table 2, these bipyridine products could not be formed using our previous approach from $\mathrm{C}-\mathrm{H}$ precursors. ${ }^{10}$ The moderate yields obtained approximate those in our previous report involving 2,2'-bipyridine couplings, and further investigations into the mechanism of this process are ongoing. ${ }^{15}$

Finally, we developed a one-pot, net cross-electrophile coupling of two chloroazines in a stage-wise protocol involving sequential addition of reagents (Table 3). ${ }^{16}$ We hypothesized that the heteroarylphosphine could be formed in situ and reacted with the second chloroazine via a tandem $\mathrm{S}_{N}$ Ar-ligand-coupling sequence from Table 2. The four examples, $\mathbf{3 e}, \mathbf{3 f}, \mathbf{3 i}$ and $\mathbf{3 y}$, in Table 3 show that pyridine-pyridine and pyridine-quinoline couplings are viable using this process forming products in reasonable yields, with $2,2^{\prime}-$, $2,4^{\prime}$ - and 4,4'-conectivity between the two heterocycles. Current efforts are focused on increasing the efficiency of these process, as well as expanding the scope to other azine-azine couplings.

Table 3. One-Pot Cross-Coupling of Chloroazines ${ }^{a}$

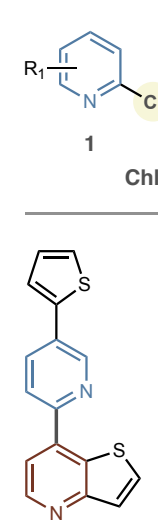

3e, $67 \%$

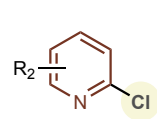

$1^{\prime}$

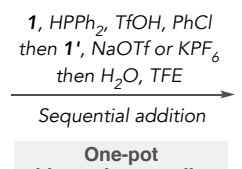<smiles>COc1cc2nccc(-c3ccccn3)c2cc1OC</smiles>

3f, $47 \%^{b}$ chloroazine coupling

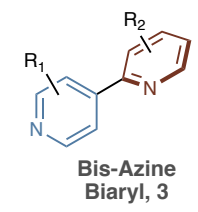

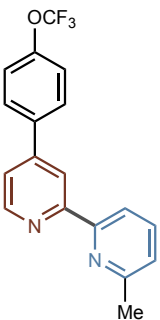

$3 y, 43 \%$

${ }^{a}$ Isolated yields are reported. ${ }^{b} \mathrm{TFE} /$ Toluene $1: 1$ used in the final stage.

In summary, we have developed an alternative strategy to form bis-azine biaryls by coupling azinylphosphines with chloroazines. The reaction proceeds via a tandem $\mathrm{S}_{N} \mathrm{Ar}$-ligand-coupling sequence, and the heteroaryl phosphines are bench-stable solids that are prepared themselves from chloroazines in a separate step. A diverse set of bis-azine biaryl products can be formed, including substitution patterns such as 2,2'-bipyridines, that are challenging for traditional metal-catalyzed approaches. Abundant chloroazines, simple protocols and valuable bis-azine biaryl products make this approach useful for medicinal chemists.

\section{ASSOCIATED CONTENT}

The Supporting Information is available free of charge on the ACS Publications

website.

Experimental procedures and spectral data (PDF)

\section{AUTHOR INFORMATION}

\section{Corresponding Author}

*andy.mcnally@colostate.edu

\section{ORCID}

Benjamin T. Boyle: 0000-0002-9360-2179

Michael C. Hilton: 0000-0001-5734-3547

Andrew McNally: 0000-0002-8651-1631

\section{Funding Sources}

This work was supported by The National Institutes of Health (NIGMS) under award number R01 GM124094.

\section{REFERENCES}

(1) (a) Liu, J.; Pan, S.; Hsieh, M. H.; Ng, N.; Sun, F.; Wang, T.; Kasibhatla, S.; Schuller, A. G.; Li, A. G.; Cheng, D.; et al. Targeting Wnt-Driven Cancer through the Inhibition of Porcupine by LGK974. PNAS 2013, 110, 20224-20229. (b) Roecker, A. J.; Mercer, S. P.; Schreier, J. D.; Cox, C. D.; Fraley, M. E.; Steen, J. T.; Lemaire, W.; Bruno, J. G.; Harrell, C. M.; Garson, S. L.; et al. Discovery of 5"-Chloro-N-[(5,6-Dimethoxypyridin-2Yl)Methyl]-2,2':5',3"-Terpyridine-3'-Carboxamide (MK-1064): A Selective Orexin 2 Receptor Antagonist (2-SORA) for the Treatment of Insomnia. ChemMedChem 2014, 9, 311-322. (c) Fu, P.; Wang, S.; Hong, K.; Li, X.; Liu, P.; Wang, Y.; Zhu, W. Cytotoxic Bipyridines from the MarineDerived Actinomycete Actinoalloteichus Cyanogriseus WH1-2216-6. $J$. Nat. Prod. 2011, 74, 1751-1756. (d) Fletcher, N. C. Chiral 2,2'-Bipyridines: Ligands for Asymmetric Induction. J. Chem. Soc., Perkin Trans. 1 2002, 0 , 1831-1842. (e) Kaes, C.; Katz, A.; Hosseini, M. W. Bipyridine: The Most Widely Used Ligand. A Review of Molecules Comprising at Least Two 2,2'-Bipyridine Units. Chem. Rev. 2000, 100, 3553-3590. (f) Roberts, J. M.; Fini, B. M.; Sarjeant, A. A.; Farha, O. K.; Hupp, J. T.; Scheidt, K. A. Urea Metal-Organic Frameworks as Effective and Size-Selective Hydrogen-Bond Catalysts. J. Am. Chem. Soc. 2012, 134, 3334-3337. (g) Suh, M. P.; Cheon, Y. E.; Lee, E. Y. Syntheses and Functions of Porous Metallosupramolecular Networks. Coordination Chemistry Reviews 2008, 252, 1007-1026. (h) Corma, A.; García, H.; Llabrés i Xamena, F. X. Engineering Metal-Organic Frameworks for Heterogeneous Catalysis. Chem. Rev. 2010, 110, 4606-4655. (i) Newkome, G. R.; Patri, A. K.; Holder, E.; Schubert, U. S. Synthesis of 2,2'-Bipyridines: Versatile Building Blocks for Sexy Architectures and Functional Nanomaterials. European Journal of Organic Chemistry 2004, 2004, 235-254.

(2) Campeau, L.-C.; Fagnou, K. Applications of and Alternatives to $\pi$ Electron-Deficient Azine Organometallics in Metal Catalyzed Cross-Coupling Reactions. Chem. Soc. Rev. 2007, 36, 1058-1068.

(3) (a) Colombe, J. R.; Bernhardt, S.; Stathakis, C.; Buchwald, S. L.; Knochel, P. Synthesis of Solid 2-Pyridylzinc Reagents and Their Application in Negishi Reactions. Org. Lett. 2013, 15, 5754-5757. (b) Yamamoto, Y.; Azuma, Y.; Mitoh, H. General Method for Synthesis of Bipyridines: Palladium Catalyzed Cross-Coupling Reaction of Trimethylstannyl-Pyridines with Bromopyridines. Synthesis 1986, 1986, 564-565. (c) Blakemore, D. C.; Marples, L. A. Palladium(0)-Catalysed Cross-Coupling of 2-Trimethylsilylpyridine with Aryl Halides. Tetrahedron Letters 2011, 52, 41924195.

(4) Brown, D. G.; Boström, J. Analysis of Past and Present Synthetic Methodologies on Medicinal Chemistry: Where Have All the New Reactions Gone? J. Med. Chem. 2016, 59, 4443-4458.

(5) A Scifinder search of the CAS database for pyridyl boronic acids (all isomers) resulted in 2686 commercially available versus 747322 commercially available chloropyridines. 
(6) Cox, P. A.; Reid, M.; Leach, A. G.; Campbell, A. D.; King, E. J.; Lloyd-Jones, G. C. Base-Catalyzed Aryl-B $(\mathrm{OH})_{2}$ Protodeboronation Revisited: From Concerted Proton Transfer to Liberation of a Transient Aryl Anion. J. Am. Chem. Soc. 2017, 139, 13156-13165.

(7) J.-P. Finet, in Ligand Coupling Reactions with Heteroaromatic Compounds, Vol. 18 (Pergamon, 1998), chap. 4

(8) (a) Molander, G. A.; Canturk, B.; Kennedy, L. E. Scope of the Suzuki-Miyaura Cross-Coupling Reactions of Potassium Heteroaryltrifluoroborates. J. Org. Chem. 2009, 74, 973-980. (b) Knapp, D. M.; Gillis, E. P.; Burke, M. D. A General Solution for Unstable Boronic Acids: SlowRelease Cross-Coupling from Air-Stable MIDA Boronates. J. Am. Chem. Soc. 2009, 131, 6961-6963. (c) Lennox, A. J. J.; Lloyd-Jones, G. C. Selection of Boron Reagents for Suzuki-Miyaura Coupling. Chem. Soc. Rev. 2013, 43, 412-443.

(9) (a) Markovic, T.; Rocke, B. N.; Blakemore, D. C.; Mascitti, V.; Willis, M. C. Pyridine Sulfinates as General Nucleophilic Coupling Partners in Palladium-Catalyzed Cross-Coupling Reactions with Aryl Halides. Chem. Sci. 2017, 8, 4437-4442. (b) Markovic, T.; Murray, P. R. D.; Rocke, B. N.; Shavnya, A.; Blakemore, D. C.; Willis, M. C. Heterocyclic Allylsulfones as Latent Heteroaryl Nucleophiles in Palladium-Catalyzed Cross-Coupling Reactions. J. Am. Chem. Soc. 2018, 140, 15916-15923.

(10) Hilton, M. C.; Zhang, X.; Boyle, B. T.; Alegre-Requena, J. V.; Paton, R. S.; McNally, A. Heterobiaryl Synthesis by Contractive C-C Coupling via P(V) Intermediates. Science 2018, 362, 799-804.

(11) We have found that $N$-aryl amides are generally compatible with $\mathrm{Tf}_{2} \mathrm{O}$ whereas $N$-alkyl amides can react competitively with azines.

(12) Barbe, G.; Charette, A.B., Highly Chemoselective Metal-Free Reduction of Tertiary Amides. J. Am. Chem. Soc. 2008, 130, 18-19.

(13) Terrier, F., Ed. In Modern Nucleophilic Aromatic Substitution; Wiley-VCH: Weinheim, Germany, 2013.

(14) Mečiarová, M.; Toma, S.; Loupy, A.; Horváth, B. Synthesis of Phosphonium Salts-Phosphine Structure and Inorganic Salts Effects. Phosphorus, Sulfur, and Silicon and the Related Elements 2007, 183, 2133.

(15) A set of guidelines and limitations for this coupling procedure are detailed in the Supporting Information.

(16) (a) Ackerman, L. K. G.; Lovell, M. M.; Weix, D. J. Multimetallic Catalysed Cross-Coupling of Aryl Bromides with Aryl Triflates. Nature 2015, 524, 454-457. (b) Everson, D. A.; Weix, D. J. Cross-Electrophile Coupling: Principles of Reactivity and Selectivity. J. Org. Chem. 2014, 79, 4793-4798. 
Insert Table of Contents artwork here

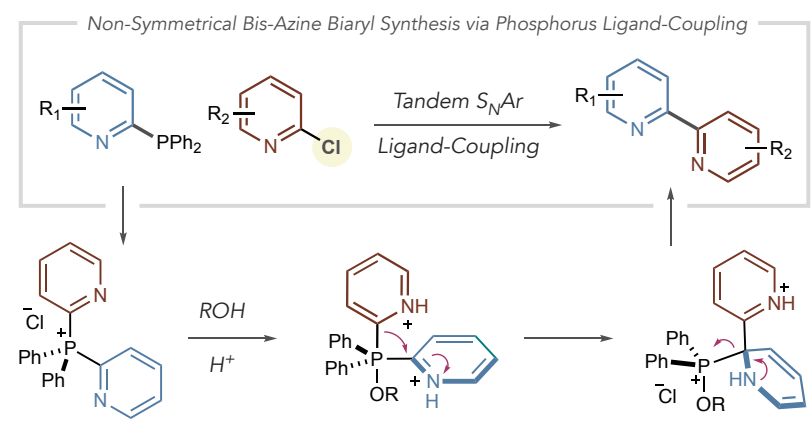

